\title{
Avaliação de dois métodos para condicionamento e coleta de sêmen em quatro espécies do gênero Mazama $^{1}$
}

\author{
Luciana D. Rola ${ }^{2 *}$, Eveline S. Zanetti² e José Maurício B. Duarte ${ }^{2}$
}

\begin{abstract}
Rola L.D., Zanetti E.S. \& Duarte J.M.B. 2012. [Assessment of two methods for conditioning and semen collection in four species of Mazama genus.] Avaliação de dois métodos para condicionamento e coleta de sêmen em quatro espécies do gênero Mazama. Pesquisa Veterinária Brasileira 32(7):658-662. Núcleo de Pesquisa e Conservação de Cervídeos, Departamento de Zootecnia, Faculdade de Ciências Agrárias e Veterinárias, Universidade Estadual Paulista, Via de Acesso Prof. Paulo Donato Castellane s/n, São Paulo, SP 14884-900, Brazil. E-mail: lanakauz@gmail.com

The development of noninvasive techniques for obtaining semen from deer facilitates the creation of genome banks, which are important tools for ex situ and in situ conservation. This study aimed to establish a noninvasive method of semen collection and compare two techniques of collection in four species of the genus Mazama: M. americana, M. gouazoubira, M. nana and M. nemorivaga. To achieve this, 6 males (M) and 2 females (F) of the species M. Americana, $3 \mathrm{M}$ and $2 \mathrm{~F}$ of M. gouazoubira, $1 \mathrm{M}$ and $1 \mathrm{~F}$ of M. nana and $2 \mathrm{M}$ and $1 \mathrm{~F}$ of M. nemorivaga were used. For each technique tested, a period of habituation to animal handling was conducted; then, the two conditioning techniques and collection were evaluated. In the first, a female in estrus was used with lateral deviation of the penis to an artificial vagina (FEDL), yielding collection from $50 \%$ of the males (100\% from M. gouazoubira and 50\% from M. americana), with no ejaculate from the remaining species. In the second technique, using a taxidermized dummy with urine from females in estrus (MUFE), no semen collection was possible. During all stages, male behavior was observed regarding the time of interest and approximation, the "Flehmen" response, the act of sniffing or licking, exposure of the penis, erection, number of false mounts, attempts at copulation and the occurrence of aggression between the deer.
\end{abstract}

INDEX TERMS: Semen, cervids, Mazama, conditioning.

RESUMO.- 0 desenvolvimento de técnicas não invasivas para a obtenção de sêmen de cervídeos facilita a criação de bancos genômicos, que são importantes instrumentos para a conservação ex situ e in situ. Este trabalho teve como objetivo criar uma metodologia não-invasiva de coleta de sêmen e comparar duas técnicas de coleta em quatro espécies do gênero Mazama: M. americana, M. gouazoubira, M. nana e M. nemorivaga. Para tanto, foram utilizados seis machos (M) e duas fêmeas (F) da espécie M. americana, $3 \mathrm{M}$ e $2 \mathrm{~F}$ de $M$.

\footnotetext{
${ }^{1}$ Recebido em 13 de janeiro de 2012.

Aceito para publicação em 6 de março de 2012.

${ }^{2}$ Núcleo de Pesquisa e Conservação de Cervídeos, Departamento de Zootecnia, Faculdade de Ciências Agrárias e Veterinárias, Universidade Estadual Paulista, Via de Acesso Prof. Paulo Donato Castellane s/n, São Paulo, SP 14884-900, Brasil. *Autor para correspondência: lanakauz@ gmail.com Os outros autores: eveline_zanetti@yahoo.com.br, barbanti@ fcav.unesp.br
}

gouazoubira, $1 \mathrm{M}$ e $1 \mathrm{~F}$ de $M$. nana e $2 \mathrm{M}$ e $1 \mathrm{~F}$ de $M$. nemorivaga. Para cada técnica testada, foi realizado um período de habituação dos animais ao manejo. Em seguida, duas técnicas de condicionamento e coleta foram avaliadas. $\mathrm{Na}$ primeira delas foi utilizada uma fêmea em estro com desvio lateral do pênis para vagina artificial (FEDL), obtendo-se a coleta de $50 \%$ dos indivíduos $(100 \%$ dos machos de M. gouazoubira e $50 \%$ dos machos de M. americana), não obtendo ejaculados das demais espécies. Na segunda técnica, utilizando um manequim taxidermizado com urina de fêmea em estro (MUFE) não foi possível a coleta de nenhum ejaculado. Em todas as fases foi observado o comportamento do macho quanto ao tempo de interesse e aproximação, reflexo de "Flehmen", ato de cheirar ou lamber, exposição do pênis, ereção, número de falsas montas, tentativas de cópula e ocorrência de agressividade entre os animais.

TERMOS DE INDEXAÇÃO: Sêmen, cervídeo, Mazama, condicionamento. 


\section{INTRODUÇÃO}

Os bancos genômicos são ferramentas cada vez mais importantes para a conservação das espécies selvagens in situ e ex situ. Para a formação destes bancos o processo de coleta de sêmen é uma etapa que merece atenção, já que pode influenciar na qualidade do ejaculado obtido e principalmente na saúde e bem estar dos animais (Krzywinski \& Bobek 1984, Pukazhenthi \& Wildt 2004).

Há três métodos principais para a coleta de sêmen em animais selvagens: eletroejaculação (EE), vagina artificial (VA) e recuperação de espermatozóides post-mortem. Nas espécies Mazama gouazoubira (Giraldi \& Duarte 2004) e Dama dama (Jabbour \& Asher 1991) já foram obtidos ejaculados utilizando uma mucosa artificial no interior da vagina de uma fêmea estrogenizada.

Devido à segurança que oferece aos coletores, a EE ainda é o principal método utilizado para a obtenção do ejaculado de animais selvagens. No entanto, este é um método que necessita da contenção química dos animais, o que acarreta em menor número de coletas, propicia maiores riscos de vida aos machos, além do risco de contaminação do ejaculado com urina e da obtenção de um ejaculado mais diluído (Wildt 1996).

0 uso da VA é considerado uma boa alternativa, pois a qualidade do ejaculado é semelhante à do coito e há redução do estresse para o animal (Asher et al. 1993), fato importante para os cervídeos, que são considerados animais muito suscetíveis ao estresse (Dias 1997), o que pode influenciar negativamente em mecanismos fisiológicos ligados a reprodução (Wildt 1996) e na qualidade seminal (VanderVoort et al. 1993). O uso da VA também permite realizar coletas sucessivas, diferentemente do que é preconizado para a coleta com EE, onde é recomendado um intervalo de uma semana entre coletas para as espécies do gênero Mazama (Duarte \& Garcia 1997).

A coleta com a VA já foi testada em algumas espécies de cervídeos através do desvio lateral do pênis durante a monta em uma fêmea estrogenizada (Mazama gouazoubira, Giraldi \& Duarte 2004) ou utilizando manequins (Cervus elaphus, Krzywinski \& Bobek 1984, Mazama gouazoubira, Giraldi \& Duarte 2004). Mesmo com a menor aceitação dos animais, a técnica de coleta com um manequim possui vantagens, como a maior segurança e agilidade do processo, tanto para o operador, quanto para os animais (Silva Filho et al. 1999) e o fato de não depender da disponibilidade de uma fêmea em estro (Giraldi \& Duarte 2004). Alguns autores sugerem que o manequim pode ser empregado como uma "armadilha espermática" para ser utilizada a campo, com animais de vida livre (Duarte \& Garcia 1997). Em $C$. elaphus, a coleta de sêmen com VA facilitou a evolução dos estudos referentes ao sêmen desta espécie, possibilitando inclusive a coleta de animais agressivos (Krzywinski \& Bobek 1984, Strzezek et al. 1985).

Como existem poucas informações a respeito do desenvolvimento de técnicas de condicionamento para a obtenção de sêmen de cervídeos, a criação de uma metodologia eficiente para esta finalidade seria importante devido à diminuição do estresse, à segurança que essa técnica oferece ao animal e às facilidades que ela traz para a construção de um banco de sêmen, tornando-se um importante instrumento a ser utilizado na conservação do gênero Mazama e de outras espécies de cervídeos ameaçadas. Assim, este trabalho teve como objetivo criar uma metodologia de condicionamento para coleta de sêmen e comparar duas técnicas de coleta (desvio lateral do pênis para vagina artificial (FEDL) e manequim taxidermizado com urina de fêmea em estro (MUFE)) em quatro espécies do gênero Mazama.

\section{MATERIAL E MÉTODOS}

Este estudo foi aprovado pelo Comitê de Ética e Bem-Estar Animal (CEBEA) da Faculdade de Ciências Agrárias e Veterinárias (FCAV), Universidade Estadual Paulista (Unesp), Jaboticabal, SP, Brasil (protocolo no 013215-07).

\section{Animais}

Foram utilizados seis machos (M) e duas fêmeas (F) da espécie Mazama americana, três M e duas F de Mazama gouazoubira, um M e uma F de Mazama nana e dois M e uma F de Mazama nemorivaga, todos adultos (3-11 anos em cativeiro) e pertencentes ao Núcleo de Pesquisa e Conservação de Cervídeos (Nupecce) (Registro IBAMA no 1/35/92/0882-5, Cadastro SISBIO no 482.508) do Departamento de Zootecnia da Universidade Estadual Paulista, Campus de Jaboticabal. Os animais foram mantidos em baias individuais ( $4 \times 4 \mathrm{~m})$, com contato auditivo e olfativo entre machos e fêmeas, e expostos a flutuações naturais do fotoperíodo. Foram alimentados diariamente (ad libitum) com ração eqüina $(12 \%$ proteína bruta, 2\% gordura, 10\% fibra; Purina Co., Paulínia, Brasil) e aproximadamente $1 \mathrm{~kg} /$ animal/dia de soja-perene (Neonotomia wightii), amoreira (Morus alba) ou alfafa (Medicago sativa). A água foi fornecida ad libitum.

\section{Técnicas de coleta}

Foram avaliadas duas técnicas de coleta: desvio lateral do pênis para uma vagina artificial (FEDL) e manequim com urina de fêmea em estro (MUFE).

Desvio lateral do pênis para uma vagina artificial (FEDL). Para a técnica FEDL, foi realizado um período de habituação de 10 dias, quando o operador permaneceu por 15 minutos no interior das baias dos animais (oferecendo a eles um alimento atrativo (banana)). Durante este período, o comportamento do macho foi observado quanto à ocorrência de agressividade, aproximação do operador e aceite de alimento diretamente da mão do operador. Com o intuito de facilitar a detecção e otimizar a utilização de todo o período de estro das fêmeas (duração aproximada de $2,2 \pm 0,3$ dias a cada 24,7 $\pm 1,2$ dias em M. gouazoubira - Pereira et al. 2006), optou-se por realizar a manipulação farmacológica do ciclo estral com o uso de $250 \mu \mathrm{g}$ de cloprostenol sódico $(1,0 \mathrm{~mL}$ Ciosin $^{\circledR}$-Intervet/Schering Plough Coopers ${ }^{\circledR}$, Brasil), por via intramuscular (Zanetti et al. 2010). O fármaco foi administrado a cada 11 dias até a detecção de quatro estros em cada uma das espécies. As fêmeas foram monitoradas a cada seis horas para a detecção do início (ocorrido entre 48-72 horas após a aplicação em M. gouazoubira - Zanetti et al. 2010) e fim do estro (ocorrido entre 86-109 horas após a aplicação em M. gouazoubira - Zanetti et al. 2010). Também durante o período de estro, foram realizadas coletas de urina das fêmeas. Para isso, o coletor permanecia dentro da baia do animal esperando a micção e, neste momento, aproximava-se da fêmea para a coleta da urina com o auxilio de uma caneca. As amostras de urina foram fracionada em alíquotas de $4 \mathrm{~mL}$, armazenadas em freezer a $-20^{\circ} \mathrm{C}$ e estocadas nesta mes- 
ma temperatura até a sua posterior utilização na segunda técnica (MUFE). Após a detecção do estro, realizada por pressão exercida na região pélvica (Curlewis et al. 1988), a fêmea e o macho foram colocados em baias diferentes, uma em frente à outra, e as portas foram abertas. Durante o tempo de contato entre eles foi observado o comportamento do macho quanto ao tempo de interesse e aproximação até a fêmea, reflexo de "Flehmen", ato de cheirar ou lamber, exposição do pênis, ereção, número de falsas montas, tentativa de cópula, ocorrência de agressividade entre os animais, tempo de coleta e volume de sêmen obtido. Durante a monta, o operador aproximou-se e desviou o pênis do animal lateralmente para a VA, colhendo o ejaculado (Fig.1). 0 tempo limite para a coleta com cada animal foi de 15 minutos, e após este período ou em caso de agressão entre os animais, estes foram separados. Durante os quatro estros sincronizados, foram realizadas tentativas de coleta (8:00-18:00 horas) com os machos e fêmeas de suas respectivas espécies, com intervalos de 30 minutos entre cada um dos machos. 0 número e a freqüência diária de coletas foi limitado pelo tempo em que a fêmea se manteve em estro e pelo número de machos de cada espécie disponíveis para o experimento.

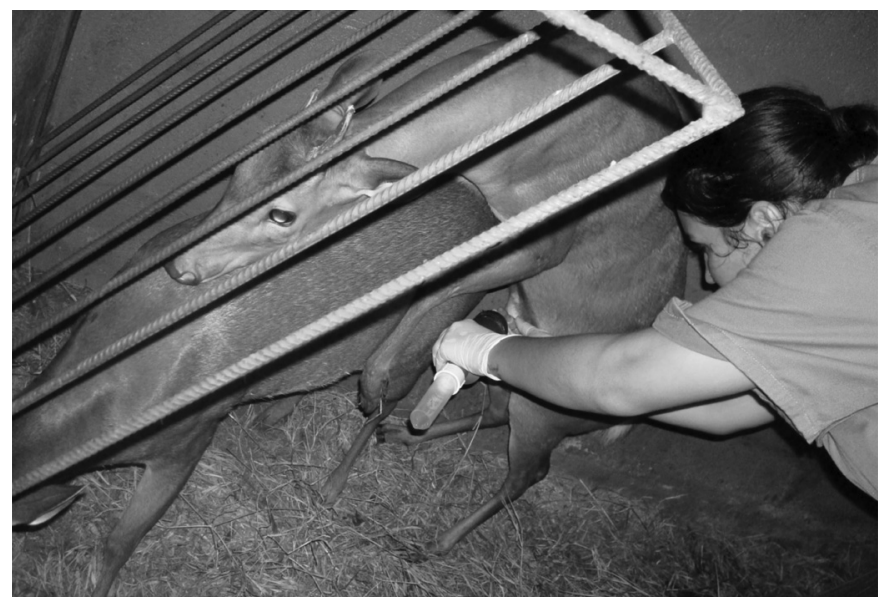

Fig.1. Macho de Mazama americana montando fêmea e operador realizando desvio lateral do pênis.

Manequim taxidermizado com urina de fêmea em estro (MUFE). Para a técnica MUFE foram utilizados somente os machos que montaram a fêmea durante a execução da primeira técnica (FEDL), pois foi pressuposto que se o macho não apresentasse libido para montar a fêmea, não seria possível treiná-lo para montar um manequim. Foram excluídos, portanto, dois machos de $M$. nemorivaga e um macho de $M$. americana. Para esta técnica foi utilizado um brete de habituação (Fig.2). 0 período de habituação foi de 15 dias, durante o qual o operador encaminhou separadamente cada indivíduo com o auxílio de uma parede móvel até o brete de habituação (Fig.2), onde permaneciam por 10 minutos. Foi oferecido alimento atrativo (banana) para todos os animais na entrada do brete e, para as fêmeas, o alimento também foi oferecido na janela do fundo do brete (Fig.2A), para que fossem atraídas a entrar no local. Após a sua entrada, a saída do brete foi ocluída, na tentativa de habituá-las a permanecer dentro do brete. 0 intuito de utilizar o brete com a fêmea em seu interior foi uma tentativa de atrair os machos e associar este local com a cópula, para que, posteriormente, a fêmea fosse substituída pelo manequim. Após o período de habituação, esta técnica foi realizada em três etapas seqüenciais. Para isto, uma fêmea de cada espécie foi estrogeinizada com a administração de 0,7-1,0mg de benzoato de estradiol (Estrogin ${ }^{\circledR}$, FarmaVet $^{\circledR}$, São Paulo, Brasil), por via intramuscular, para que o tempo de duração do estro fosse maior do que o estro

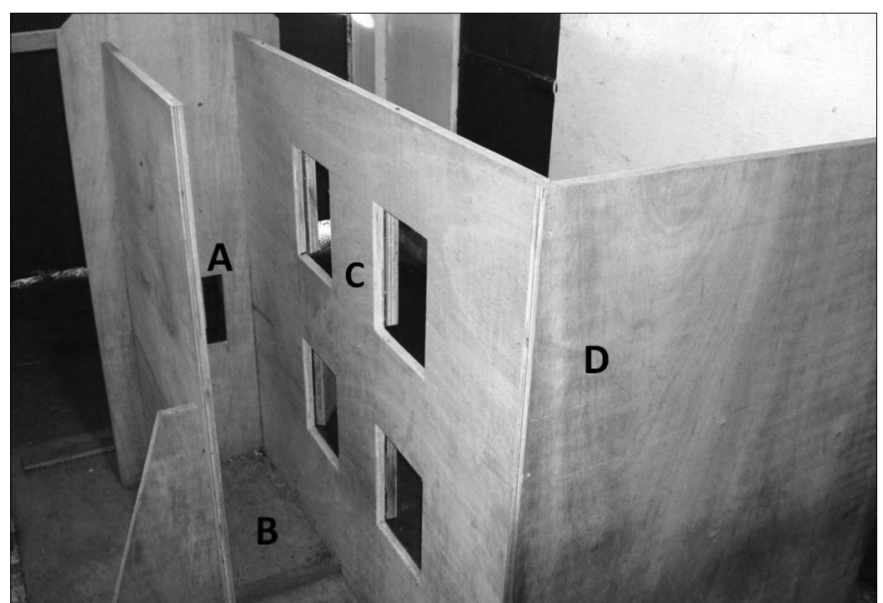

Fig.2. Brete para habituação e condicionamento dos animais ao manejo de coleta de sêmen com a técnica MUFE (manequim taxidermizado com urina de fêmea em estro). (A) Local onde foi oferecido alimento atrativo às fêmeas. (B) Local onde o manequim foi posicionado dentro do brete. (C) Janelas laterais, por onde o operador tem acesso ao animal para o desvio lateral do pênis. (D) parede falsa, atrás de onde o operador estaria posicionado.

natural ou sincronizado e permitisse a realização das três etapas da técnica de MUFE: etapa (1) o macho foi encaminhando até o brete (com auxílio de uma parede móvel), onde estava a fêmea em estro e a cópula foi permitida; etapa (2) idem etapa 1, mas foi realizado o desvio lateral do pênis e a coleta do sêmen com a VA; etapa (3) a fêmea foi substituída por um manequim taxidermizado com urina (que estava armazenada a -20ำ) de fêmea em estro no dorso (Fig.2B). Em seguida, o macho foi encaminhado até o local e, quando montasse o manequim, o desvio lateral do pênis seria realizado através das janelas laterais (Fig.2C), uma vez que o operador estaria posicionado atrás da parede falsa do brete (Fig.2D). 0 período de intervalo entre as etapas foi de 30 minutos, e em todas as etapas o tempo limite para que ocorresse a cópula (etapa 1) ou coleta (etapas 2 e 3 ) foi de 10 minutos. Após os 10 minutos, ou no caso de agressão entre os animais (nas etapas $1 \mathrm{e}$ 2 ), estes foram separados e encaminhados de volta às suas baias. 0 comportamento dos machos foi observado em todas as etapas quanto ao reflexo de Flehmen, ato de cheirar ou lamber, exposição do pênis, ereção, número de falsas montas, tentativa de cópula, ocorrência de agressividade entre os animais ou entre macho e manequim, tempo de coleta e volume de sêmen obtido. Esta técnica foi realizada duas vezes com cada um dos machos. Para a execução da técnica com o macho de $M$. nana foi utilizada uma das fêmeas de M. gouazoubira, com o intuito de não submeter a única fêmea de M. nana do plantel à administração de benzoato de estradiol devido a urgente necessidade de reprodução desta espécie em cativeiro. A existência de híbridos entre estas duas espécies demonstra a interação sexual em cativeiro (J.M.B. Duarte, comunicação pessoal).

\section{Análise estatística}

Os dados estão apresentados como porcentagem de ocorrência e média \pm desvio padrão da média (DPM).

\section{RESULTADOS}

Com a técnica de FEDL foram obtidos ejaculados de 50\% dos indivíduos deste experimento (totalizando 53 ejaculados), com coleta de 100\% dos machos de Mazama goua- 
zoubira e $50 \%$ dos indivíduos de M. americana. 0 ejaculado foi obtido em todas as vezes que o operador tentou a coleta dos machos que apresentaram o estímulo de montar a fêmea, com exceção do macho de $M$. nana, que não permitiu a aproximação total do operador durante a habituação e também para a realização da coleta, inviabilizando a obtenção do ejaculado. Não foi observado nenhum comportamento agressivo com o operador durante as coletas.

A ausência de ejaculados na espécie $M$. nemorivaga está relacionada à falta de interesse e libido dos machos, bem como o distanciamento da fêmea no momento em que os machos tentavam se aproximar. No caso da espécie $M$. americana, os ejaculados não foram obtidos dos machos que mostraram menor interesse sexual. Nos machos com menor interesse houve agressão em 52,4\% das sessões de coleta, enquanto com aqueles que apresentaram maior interesse e permitiram a coleta, a agressão foi observada em apenas $9 \%$ das sessões. Destes últimos, o tempo médio de aproximação foi de 27 segundos, enquanto nos machos sem interesse pelas fêmeas, este tempo foi decorrente da aproximação da fêmea para agredir o macho, sendo em média de 2 minutos.

Para as espécies M. gouazoubira, M. americana, M. nemorivaga e $M$. nana, o tempo médio de aproximação foi 10 seg \pm 3 seg, $1 \mathrm{~min}$ e $19 \mathrm{seg} \pm 1 \mathrm{~min}, 3 \mathrm{~min}$ e $26 \mathrm{seg} \pm 48 \mathrm{seg}$ e 13 seg \pm 10 seg, respectivamente. A média do volume de sêmen coletado foi de $0,06 \pm 0,025 \mathrm{~mL}$ para M. gouazoubira e $0,13 \pm 0,068 \mathrm{~mL}$ para M. americana.

As tentativas de coleta com esta técnica foram realizadas durante todo o período que as fêmeas permaneceram receptivas nas quatro sincronizações de estro, variando de 7 a 13 sessões de coleta com cada um dos machos e os comportamentos observados nos animais que montaram as fêmeas foram a exposição do pênis, ereção do pênis e o ato de cheirar/lamber a posterior da fêmea.

Não foram obtidos ejaculados dos animais com a técnica MUFE, pois não foi observado interesse sexual dos machos em relação ao manequim, e para $M$. nana não houve sequer o reconhecimento entre o macho e a fêmea de $M$. gouazoubira, ou da urina de fêmea em estro desta espécie, sugerindo uma incompatibilidade sexual entre o casal. Os comportamentos observados durante as coletas foram o ato de cheirar/lamber o manequim (na área em que a urina foi colocada), o reflexo de Flehmen e a agressão de alguns machos em relação ao operador e ao manequim.

\section{DISCUSSÃO E CONCLUSÃO}

Nossos resultados indicam que é possível a coleta de sêmen das espécies Mazama gouazoubira e M. americana, utilizando a técnica FEDL, assim como já observado por Giraldi \& Duarte (2004), que obtiveram ejaculados de $75 \%$ dos animais da espécie M. gouazoubira, utilizando essa mesma técnica. No entanto, estes autores não realizaram um período de adaptação antes do início do experimento, o que pode ter resultado em menores índices de coleta.

A ausência de comportamento agressivo com o operador durante as coletas demonstra que o período de habituação anterior à técnica foi efetivo e que a FEDL é uma técnica segura para coleta de sêmen destes animais. A agressivida- de das fêmeas em relação a alguns machos da espécie $M$. americana parece ter sido desencadeada pelo menor interesse sexual do macho. No entanto, com o decorrer das sessões estes machos apresentaram maior interação com as fêmeas e, possivelmente, o sucesso de coleta com eles dependeria de um maior tempo experimental.

É provável que o sucesso da técnica FEDL dependa basicamente de dois fatores: 1) fêmeas e machos que permitam a aproximação do operador e, para isso, o período de adaptação parece ser decisivo; 2) o macho deve ter interesse sexual e, quanto maior a sua libido, maior a probabilidade de sucesso na coleta.

Os volumes dos ejaculados coletados corroboram com a descrição de que os volumes obtidos com o uso da VA são menores do que aqueles obtidos com a eletroejaculação, que variam de 0,2 a $0,4 \mathrm{~mL}$ para $M$. americana e 0,1 a $0,7 \mathrm{~mL}$ para M. gouazoubira (Duarte \& Garcia 1997). Utilizando a VA para a espécie M. gouazoubira, Giraldi \& Duarte (2004) obtiveram uma média de volume de $0,11 \mathrm{~mL}$, valor maior do que o encontrado em nosso experimento. 0 menor volume encontrado no presente experimento ocorreu devido à perda de ejaculado no adaptador da VA, fato que foi notado após algumas sessões de coleta e que se repetiu na maioria das amostras coletadas posteriormente. Este fato chama a atenção para a necessidade da utilização de uma VA melhor adaptada às necessidades da espécie ou para um melhor posicionamento da VA no momento da cópula, já que a VA utilizada foi desenvolvida para ovinos e é possível que não se adapte perfeitamente para o uso em cervídeos, conforme anteriormente relatado em outras espécies selvagens (Bravo et al. 1997, Mosaferi et al. 2005, Giuliano et al. 2008).

A opção de realizar a técnica MUFE somente com os indivíduos que montaram a fêmea na técnica FEDL justifica-se pelo fato de que se o animal não apresentasse interesse pela fêmea viva, não seria possível seu treinamento e ele dificilmente apresentaria interesse pelo manequim. No entanto, nenhum ejaculado foi obtido com esta técnica, pois não foi observado libido dos machos em relação ao manequim. Para espécies domésticas (Foote 1974) e algumas espécies selvagens como lhamas (Lichtenwalner et al. 1996), alpacas (Bravo et al. 1997) e cervídeos (Krzywinski \& Bobek 1984, Giraldi \& Duarte 2004), o comportamento das coletas com o manequim foi similar àquele apresentado com uma fêmea, entretanto, para lhamas (Lichtenwalner et al. 1996) e M. gouazoubira (Giraldi \& Duarte 2004), foi necessária a presença de uma fêmea receptiva para estimular a monta dos machos no manequim.

Em machos de ungulados domésticos, os atos de cheirar e lamber a fêmea são os padrões de comportamento mais freqüentes, demonstrando a importância da comunicação química entre os animais, realizada através da olfação (Alexander et al. 1974). Por este motivo, foi estabelecido o uso de urina de fêmea em estro no manequim. Além disso, experimentos com animais domésticos sugerem que as informações essenciais para que ocorra a resposta sexual do macho são a forma do objeto semelhante à fêmea e sua imobilidade, lembrando a receptividade (Alexander et al. 1974). Entretanto, o uso da urina de fêmea em estro no manequim taxidermizado e as tentativas de associá-lo 
a fêmea não foram suficientes para que os machos apresentassem o comportamento sexual característico na etapa três da técnica do MUFE. Corroborando com as observações feitas para bovinos domésticos de que qualquer objeto tridimensional semelhante à região posterior da fêmea poderia atuar como estímulo sexual (Hafez \& Bouissou 1975), Krzywinski \& Bobek (1984), durante sete anos de experimento, obtiveram ejaculados de $30 \%$ dos animais (5 animais), da espécie Cervus elaphus, após um treinamento que consistia na alocação dos machos junto ao manequim com urina de fêmea em estro.

Como foi descrito por Hafez \& Bouissou (1975), é possível que os animais exerçam seu comportamento sexual por meio do aprendizado associativo. 0 encaminhamento dos machos até um local específico (brete de habituação) e a divisão da técnica MUFE em três etapas, teve por objetivo esta associação. Além disso, outras pesquisas já demonstraram que a maioria garanhões monta e ejacula em manequins com o mínimo de treinamento (Conboy 1992). No entanto, isto não foi verificado no presente experimento, talvez porque a metodologia de MUFE foi testada somente duas vezes com cada macho, devido à dificuldade de se manter as fêmeas destas espécies constantemente em estro. Nos animais domésticos a freqüência de treinamento pode ser maior e é facilitada pelo maior número de animais disponíveis e a possibilidade de manipulação direta (utilizar cabrestos para imobilizar fêmeas ou desviar machos das fêmeas para um manequim), o que muitas vezes dispensa a necessidade da presença de uma fêmea em estro (Silva Filho et al. 1999).

Diferentemente do ocorrido na técnica de FEDL, alguns machos agrediram o operador durante a realização da técnica de MUFE, e talvez isso se relacione ao fato do período de habituação entre animal-operador não ter sido realizado antes das coletas.

Assim, a técnica MUFE mostrou-se inviável para as quatro espécies avaliadas, nas condições do presente experimento, enquanto é provável que a viabilidade da técnica FEDL dependa basicamente do comportamento individual dos machos, apesar de variações interespecíficas terem sido observadas. Machos mansos e que apresentem libido com relação às fêmeas em cativeiro, possivelmente se adequarão à técnica, independentemente da espécie a que pertencem. Portanto, o presente estudo mostrou que a técnica de coleta de sêmen com o uso de uma fêmea em estro (FEDL) pode ser utilizada para as espécies Mazama americana e M. gouazoubira, colaborando para a elaboração mais fácil e segura de um banco de germoplasma.

Agradecimentos.- Agradecemos o apoio financeiro concedido pela Fundação de Apoio à Pesquisa do Estado de São Paulo (FAPESP, Processo 03/07904-0 e 2007/02593-8) e pelo Conselho Nacional de Desenvolvimento Científico e Tecnológico (CNPq). Especial agradecimento aos integrantes do "Núcleo de Pesquisa e Conservação de Cervídeos" (Nupecce-Unesp) pelo auxílio com os animais.

\section{REFERÊNCIAS}

Alexander G., Signoret F.P. \& Hafez E.S.E. 1974. Sexual and maternal behavior, p.222-254. In: Hafez E.S.E. (Ed.), Reproduction in Farm Animals. $3^{\text {rd }}$ ed. Bailliére Tindall, London.
Asher G.W., Fisher M.W., Fennessy P.F., Mackintosh C.G., Jabbour H.N. \& Marrow C.J. 1993. Oestrus synchronization, semen collection and artificial insemination of farmed Red Deer (Cervus elaphus) and Fallow Deer (Dama dama). Anim. Reprod. Sci. 33:241-265.

Bravo P.W., Flores U., Garnica J. \& Ordofiez C. 1997. Collection of semen and artificial insemination of alpacas. Theriogenology 47:619-626.

Conboy H.S. 1992. Training the novice stallion for artificial breeding. Vet. Clin. North Am., Equine Pract. 8:101-109

Curlewis J.D., Loudon A.S.I. \& Coleman A.P.M. 1988. Oestrous cycles and the breeding season of the Père David's deer hind (Elaphurus davidianus). J. Reprod. Fertil. 82:119-126.

Dias J.L.C. 1997. Miopatia de captura, p.172-179. In: Duarte J.M.B. (Ed.), Biologia e Conservação de Cervídeos Sul-americano: Blastocerus, Ozotocerus e Mazama. Funep, Jaboticabal.

Duarte J.M.B. \& Garcia J.M. 1997. Tecnologia da reprodução para propagação e conservação de espécies ameaçadas de extinção, p.228-238. In: Duarte J.M.B. (Ed.), Biologia e Conservação de Cervídeos Sul-americano: Blastocerus, Ozotocerus e Mazama. Funep, Jaboticabal.

Foote R.H. 1974. Artificial insemination, p.409-431. In: Hafez E.S.E. (Ed.), Reproduction in Farm Animals $3^{\text {rd }}$ ed. Lea and Febiger, Philadelphia.

Giraldi T. \& Duarte J.M.B. 2004. Implantação e avaliação de métodos não invasivos para colheita de sêmen de veado-catingueiro (Mazama gouazoubira). Anais XVIII Congresso da Associação Brasileira de Veterinários de Animais Selvagens, Jaboticabal, SP, p.83. (Resumo)

Giuliano S., Director A., Gambarotta M., Trasorras V. \& Miragaya M. 2008. Collection method, season and individual variation on seminal characteristics in the llama (Lama glama). Anim. Reprod. Sci. 104:359-369.

Hafez E.S.E. \& Bouissou M.F. 1975. The behaviour of cattle, p.203-245. In: Hafez E.S.E. (Ed.), The Behaviour of Domestic Animals $3^{\text {rd }}$ ed. Bailliére Tindall, London.

Jabbour H.N. \& Asher G.W. 1991. Artificial breeding of farmed fallow deer (Dama dama), p.485-491. In: Renecker L.A. \& Hudson R.J. (Eds), Wildlife Production: Conservation and sustainable development. University of Alasca Fairbanks, Fairbanks.

Krzywinski A. \& Bobek B. 1984. Semen collection from red deer males with a dummy. Acta Zoologica Fennica 171:175-178.

Lichtenwalner A.B., Woods G.L. \& Weber J.A. 1996. Seminal collection, seminal characteristics and pattern of ejaculation in llamas. Theriogenology 46:293-305.

Mosaferi S., Niasari-naslaji A., Abarghani A., Gharahdaghi A.A. \& Gerami A. 2005. Biophysical and biochemical characteristics of bactrian camel semen collected by artificial vagina, Theriogenology 63:92-101.

Pereira R.J.G., Polegato B.F., Souza S., Negrão J.A. \& Duarte J.M.B. 2006. Monitoring ovarian cycles and pregnancy in brown brocket deer (Mazama gouazoubira) by measurement of fecal progesterone metabolites. Theriogenology 65(2):387-399.

Pukazhenthi B. \& Wildt D. 2004. Which reproductive technologies are most relevant to studying, managing and conserving wildlife? Reprod. Fertil. Develop. 16:33-46.

Silva Filho J.M., Valle G.R., Viana W.S., Vianna L.R. \& Palhares M.S. 1999. Utilização de manequim para coleta de sêmen eqüino e sua influência sobre características reprodutivas do garanhão. Arq. Bras. Med. Vet. Zootec. 51(5):499-504.

Strzezek J., Krzywinski A. \& Swidowicz K. 1985. Seasonal changes in the chemical composition of red deer (Cervus elaphus) semen. Anim. Reprod. Sci. 9:195-204.

VanderVoort C.A., Neville L.E., Tollner T.L. \& Field L.P. 1993. Noninvasive semen collection from an adult orangutan. Zoo Biology 12(3):257-265.

Wildt D.E. 1996. Male reproduction: assessment, management, and control of fertility, p.129-150. In: Kleiman D.G., Allen M.E., Thompson K.V. \& Lumpkin S. (Eds), Wild Mammals in Captivity. University of Chicago Press, Chicago.

Zanetti E.S., Polegato B.F. \& Duarte J.M.B. 2010. Comparison of two methods of synchronization of estrus in brown brocket deer (Mazama gouazoubira). Animal Reproduction Science 117(3-4):266-274. 